\title{
Diferencias de sexo en estrategias de aprendizaje de estudiantes online
}

\section{Sex differences in learning strategies of online students}

\author{
Mercedes Romero Rodrigo ${ }^{1}$, Ángel San Martín Alonso², José Peirats Chacón ${ }^{3}$ \\ ${ }^{1}$ Universidad Internacional de Valencia, España (mmromero@universidadviu.com) \\ ${ }^{2}$ Departamento Didáctica y Organización Escolar, Universitat de València, España (angel.sanmartin@uv.es) \\ ${ }^{3}$ Departamento Didáctica y Organización Escolar, Universitat de València, España (jose.peirats@uv.es)
}

Recibido el 5 de mayo de 2018; revisado el 8 de octubre de 2018; aceptado el 22 de octubre de 2018; publicado el 1 de diciembre de 2018

\section{RESUMEN:}

Analizamos aquí las estrategias de aprendizaje empleadas por estudiantes universitarios, de modalidad online, en función del género. En primer lugar, se realiza una exhaustiva revisión de publicaciones que comparten el objeto de estudio, la mayoría de ellas difundidas en la última década y señalando los principales hallazgos de estas investigaciones. El objetivo del estudio es analizar tanto las estrategias empleadas como desvelar semejanzas y diferencias con respecto a modalidades presenciales.

Metodológicamente optamos por realizar un diseño multimétodo, en el que el análisis cualitativo tiene como finalidad contribuir a la evaluación e interpretación de los resultados de tipo cuantitativo. Los instrumentos de recogida de información han sido el Cuestionario de Evaluación de las Estrategias de Aprendizaje de los Estudiantes Universitarios (CEVEAPEU), análisis documental de reglamentos, memorias e informes de la universidad, revisión de aulas virtuales, guías didácticas y un seminario asíncrono en el que se les solicitaba a los estudiantes una valoración acerca de sus estrategias de aprendizaje.

Entre los resultados obtenidos cabe destacar las semejanzas contrastadas si los resultados son comparados con los apuntados en estudios presenciales, donde las mujeres muestran un perfil estratégico más sólido en comparación con sus compañeros. Esto es, planifican y aprovechan más el tiempo de estudio, presentan una motivación intrínseca por el aprendizaje, tienen una marcada preferencia por realizar trabajos y estudiar con otros compañeros, comentar dudas y pedir ayuda tanto a sus iguales como al profesorado. Sin embargo, en la estrategia de control de la ansiedad las mujeres obtuvieron peor resultado.

\section{PALABRAS CLAVE: UNIVERSIDAD VIRTUAL, APRENDIZAJE COMBINADO, EDUCACIÓN SUPERIOR, ESTRATEGIAS DE APRENDIZAJE.}

\section{ABSTRACT:}

We analyze here the learning strategies used by university students of online mode, according to sex. First of all, an exhaustive review of publications that share the object of study has been carried out, most of them disseminated in the last decade that indicate the main findings of these researches. The objective of the study is to analise the strategies employed and reveal similarities and differences with regard to face-to-face mode.

Methodologically, we chose to carry out a multimethod design, in which the qualitative analysis aims to contribute to the evaluation and interpretation of quantitative results. The information collection instruments have been the Questionnaire to Evaluate University Students' Learning Strategies (CEVEAPEU), documentary analysis of regulations, university reports, review of virtual classrooms, didactic guides and 
asynchronous seminar where we asked the students for an assessment about their learning strategies.

Among the results obtained, it should be noted the proven similarities if the results are compared with face-to-face studies, where women show a more solid strategic profile compared to their peers. In this regard, they plan and get more out of study time, provide an intrinsic motivation for learning, have a marked preference for doing work and study with other colleagues, discuss doubts and ask for help from both peers and teachers. However, in the anxiety control strategy women obtained worse results.

\section{KEYWORDS: VIRTUAL UNIVERSITIES, BLENDED LEARNING, HIGHER EDUCATION, LEARNING STRATEGIES.}

\section{INTRODUCCIÓN}

Como señaló Bates (2001), aunque las nuevas tecnologías han estado en un primer momento al servicio del alumnado tradicional, en la enseñanza online está emergiendo un mercado masivo en el que la tecnología asumirá una mayor relevancia en los procesos de enseñanza y aprendizaje. El crecimiento del número de estudios superiores a distancia (primer eslabón de la educación online tal y como la conocemos hoy en día) en instituciones públicas y privadas se duplicó en el último quinquenio (García, 2001), dando respuesta a necesidades formativas más allá del tiempo y el espacio, y evolucionando hacia nuevas formas y modelos, algunos de ellos basados en software libre (San Martín, Peirats y Sales, 2006), en la gamificación en entorno escolar (Raftopoulos, 2014) o en la realidad aumentada (Cabero y Marín, 2017; Gómez, 2018) entre otros. Un claro ejemplo de este crecimiento y su evolución lo podemos observar en el contexto español, donde conviven distintas modalidades de enseñanza y aprendizaje: desde la educación a distancia ya antes citada, la enseñanza y los aprendizajes abiertos, blearning o m-learning, teleformación, enseñanza virtual, formación online, entre otras.

A su vez, el estudio de las estrategias de aprendizaje es un tema de interés y relevancia actual: entre 2000-2011 se publicaron un total de 43 artículos de investigación en el que analizaban los estilos y estrategias aprendizaje, junto con el rendimiento y el logro de los estudiantes universitarios (Bahamón, Vianchá y Alarcón, 2012), si bien es cierto que todos ellos hacen referencia a modalidades presenciales de enseñanza.
La inquietud hacia el estudio y reflexión sobre las diferencias en función del género no es nueva en el nuestro ámbito, sino que viene siendo una constante en prácticamente todas las disciplinas académicas. Sin ir más lejos, un claro ejemplo de esta tendencia es el estudio recientemente publicado de Sánchez, Conde, Meroño, Arias y García (2018) sobre el éxito académico en carreras duales diferenciando por género. Podríamos explicar este interés como resultado de la toma de conciencia en lo que al cuestionamiento del orden patriarcal se refiere, en cómo se ha construido históricamente la identidad de los sujetos y el esclarecimiento de los mecanismos de dominación simbólica (Bourdieu, 2000) utilizados con el paso de los siglos. Según Scott (1986, p. 1056):

(...) género pasa a ser una forma de denotar las "construcciones culturales", la creación totalmente social de ideas sobre los roles apropiados para mujeres y hombres. Es una forma de referirse a los orígenes exclusivamente sociales de las identidades subjetivas de hombres y mujeres. Género es, según esta definición, una categoría social impuesta sobre un cuerpo sexuado ${ }^{1}$.

Por esta razón, identificar las diferencias entre género ${ }^{2}$, desnaturalizarlas y señalar cómo los estereotipos (tabla 1) pueden haber influido en la construcción de la identidad de los sujetos y, por extensión, en cómo estos se enfrentan a las situaciones de aprendizaje, nos proporcionará una visión más realista y compleja de la realidad a la que se enfrenta el estudiantado universitario. Es, por este motivo, por el que presentamos a continuación los principales hallazgos de los estudios de las diferencias en el uso de estrategias de aprendizaje.

\subsection{Estrategias de aprendizaje y sexo de los estudiantes: el estado de la cuestión}

Martín y Camarero (2001) realizaron un estudio sobre estilos, estrategias de aprendizaje y su relación con el género y las titulaciones universitarias. Para ello contaron con una muestra de 1174 estudiantes de la Universidad de Oviedo, a los que administraron los cuestionarios CHAEA (Alonso, Gallego y Honey, 1994) y ACRA (Román y Gallego, 1994). Centrándonos en los resultados sobre género y estrategias de aprendizaje, tal y como apuntaron los investigadores Martín y Camarero (2001):

\footnotetext{
${ }^{1}$ Traducción recogida de la obra de Amelang y Nasch (1990).

${ }^{2}$ Por tanto, en este trabajo, al entender que el género es una construcción social, pondremos el foco de estudio en el sexo.
} 
(...) sí parecen existir notables diferencias en relación a las estrategias de aprendizaje asociadas a las fases de adquisición y recuperación de la información de mayor empleo por parte de las mujeres, mientras que ciertas estrategias son empleadas más por los varones (...) pero que dichos datos han de ser analizados desde la interacción de los factores de género y tipo de carrera que modula las diferencias apuntadas (...) y estas diferencias pueden estar motivadas de los contenidos específicos de la tarea, que fortalecen o debilitan el uso de ciertos recursos intelectuales (p. 599).

Tabla 1. Estereotipos de género. Características asociadas al hombre y la mujer

\begin{tabular}{|c|c|c|c|}
\hline Rasgos & Roles & $\begin{array}{l}\text { Características } \\
\text { Psicológicas }\end{array}$ & Habilidades cognitivas \\
\hline \multicolumn{4}{|c|}{ Características asociadas al hombre } \\
\hline Activo & $\begin{array}{l}\text { Asume las obligaciones } \\
\text { financieras }\end{array}$ & Atlético & Analítico \\
\hline Toma de decisiones fácilmente & Cabeza de familia & Musculoso & Exacto \\
\hline Competitivo & Proveedor de la economía & Hombros anchos & Bueno para abstraerse \\
\hline Se siente superior & Líder & Corpulento & Bueno en los números \\
\hline Independiente & Manitas de la casa & $\begin{array}{l}\text { Fuerte } \\
\text { psicológicamente }\end{array}$ & $\begin{array}{l}\text { Bueno para resolver } \\
\text { problemas }\end{array}$ \\
\hline Nunca se rinde & $\begin{array}{l}\text { Iniciativa en las relaciones } \\
\text { sexuales }\end{array}$ & Buen estado físico & $\begin{array}{l}\text { Buen razonamiento } \\
\text { matemático }\end{array}$ \\
\hline $\begin{array}{l}\text { Seguro de sí mismo } \\
\text { Está bien bajo presión }\end{array}$ & $\begin{array}{l}\text { Ve los deportes en la } \\
\text { televisión }\end{array}$ & $\begin{array}{ll}\text { Robusto } \\
\text { Alto }\end{array}$ & Competencia cuantitativa \\
\hline \multicolumn{4}{|c|}{ Características asociadas a la mujer } \\
\hline Servicial & Cocina & Hermosa & Artística \\
\hline Cordial & Hace la compra & Linda, guapa & Creativa \\
\hline Emocional & Se encarga de lavar la ropa & Delicada & Expresiva \\
\hline Gentil & Le gusta la moda & Vistosa & Imaginativa \\
\hline Generosa & $\begin{array}{l}\text { Es el apoyo emocional de la } \\
\text { casa }\end{array}$ & Graciosa & Intuitiva \\
\hline \multirow{3}{*}{$\begin{array}{c}\text { Comprensiva } \\
\text { Consciente de los sentimientos de los } \\
\text { demás }\end{array}$} & Cuida de los hijos & Pequeña & \multirow{3}{*}{$\begin{array}{l}\text { Perceptiva } \\
\text { Elegante, con gusto } \\
\text { Competencia verbal }\end{array}$} \\
\hline & & Bonita & \\
\hline & Se encarga del hogar & $\begin{array}{l}\text { Atractiva } \\
\text { Voz suave }\end{array}$ & \\
\hline
\end{tabular}

Nota: traducción propia de Kite (2001, p. 563).

En la investigación participaron estudiantes de carreras técnicas (Informática de Gestión y de Sistemas), experimentales (Matemáticas y Física) y de humanidades (Derecho y Magisterio). Los resultados indicaron que existían diferencias asociadas al sexo, tanto en los estudios técnicos como en los experimentales y en humanidades, donde se apreciaba especialmente mayores puntuaciones en las escalas de adquisición y recuperación por parte de las mujeres. Si concretamos los resultados atendiendo a las carreras universitarias de los participantes, los resultados obtenidos mostraron que:

$>$ Las estudiantes de enseñanzas técnicas superaron a sus compañeros en todas las escalas (adquisición, recuperación, apoyo y codificación) apreciándose diferencias significativas por género. En concreto, los estudiantes quedaron definidos por el uso de autopreguntas, mientras que las estudiantes por el empleo de subrayados y codificaciones para el recuerdo.

$>$ Las mujeres de Matemáticas y Física (estudios experimentales) obtuvieron resultados significativos en las escalas de adquisición, codificación y recuperación. Destacó la estrategia de autoconocimiento (escala apoyo). Los hombres solo destacaron, en uso de la estrategia de aprendizaje, en el empleo de imágenes mentales en comparación con las mujeres, quedando definidos por el empleo de estrategias metacognitivas de automanejo, autoevaluación, autorregulación (escala apoyo) e intracontenido (escala codificación). En el caso de las estudiantes, ellas quedaron definidas por mayor empleo de subrayados (escala adquisición), resúmenes (escala codificación) y de presentación y orden de la respuesta escrita (escala recuperación). 
$>$ En cuanto a las estudiantes de Derecho y Magisterio (estudios de humanidades) obtuvieron puntuaciones más altas en las escalas de adquisición (estrategias de repasos en voz alta y subrayado idiosincrásico) y recuperación (presentación y orden de la respuesta escrita). Destacando el uso de la estrategia uso de la interacción social (escala apoyo) por encima del resto. En el caso de los estudiantes, en la escala de codificación destacaron, en comparación con el resto de escalas y estrategias, en la búsqueda de aplicación de los aprendizajes y las autopreguntas.

Por su parte, Cano (2000) concluyó que existía una diferencia significativa en las estrategias de aprendizaje empleadas según el sexo: las estudiantes superaron a sus compañeros en motivación, interés, ayudas para el estudio, interés y actitud ante el estudio, organización del tiempo, así como en el repaso y comprobación de los niveles de comprensión logrados. Además, su investigación constató que la variable contexto o "contextual", en este caso el tipo de carrera, actuaba como moduladora de las diferencias existentes entre géneros. A continuación, presentamos los resultados más relevantes de dicha investigación:

$>$ Las estudiantes de Física, Química, Arquitectura, Medicina (carreras de ciencias) obtuvieron mayor puntuación en la escala de autocomprobación. Esto significa que utilizaban más las estrategias como repasos mentales, preguntas antes y después de las lecturas, etc.

$>$ En cuanto a los universitarios de Psicología, Derecho, Formación del profesorado (ciencias sociales) obtuvieron puntuaciones altas en estrategias motivacionales (extrínseca y de logro).

A nivel internacional hay que destacar la investigación realizada en Argentina, en la Universidad de La Plata por Rossi, Neer, Lopetegui, y Doná (2010). Aunque el punto de partida fueran las propias diferencias existentes entre sexos y el rendimiento de los estudiantes, el objeto de estudio fue el uso de estrategias de aprendizaje en función del género. Los resultados obtenidos indicaron que las estrategias más utilizadas eran aquellas relacionadas con el apoyo al aprendizaje y hábitos de estudio.

Otra investigación que constató las diferencias entre estudiantes en función del sexo fue la de Aguilar (2010). La muestra estaba compuesta por estudiantado de nuevo ingreso y se corroboraron diferencias en los modos de procesar y transformar la información. De hecho, a excepción de la escala de procesamiento de la información, se observaron diferencias significativas en todas las demás: apoyo (actitud, motivación, administración del tiempo), orientación a la meta (selección de ideas principales, preparación de exámenes) y estrategias cognitivas (procesamiento de la información, técnicas de estudio). Esta investigación señaló, igual que lo hicieron las de Rossi et al. (2010) y García (2011), que los hombres tenían un mejor control de la escala ansiedad en comparación de las mujeres.

Da Cuña, Gutiérrez, Barón y Labajos (2014) determinaron, en otro trabajo, que no existían diferencias entre las estrategias de aprendizaje y el sexo de los estudiantes de Fisioterapia según el nivel de estudios de los progenitores. La única excepción la encontraron en las estudiantes de madres que poseían estudios medios: ellas usaban más la memorización que las compañeras con madres con estudios básicos. Además, no se observó que los alumnos tuvieran una relación significativa entre el nivel de estudios de la madre y las estrategias utilizadas. Tampoco se encontraron relaciones significativas con las estrategias y el nivel de estudios del padre.

Resultados similares arrojaron la investigación de Sepúlveda et al. (2010, 2011). Dichos estudios señalaron que existían diferencias por sexos en el rendimiento y en las estrategias de aprendizaje de los estudiantes de Farmacia y Química. En concreto, las mujeres tenían un mayor rendimiento y se caracterizaron por hacer un uso mayor de estrategias de tipo cognitivo que los hombres: las estudiantes recurrían a la realización de resúmenes y esquemas. En cuanto a la escala de autoconocimiento, solo la estrategia de elaboración (preparación de información ante la realización de una prueba o examen) fue la única con un resultado significativo si se atendía al sexo. En cuanto al subrayado y el uso de lápices de colores, del mismo modo que Martín y Camarero (2001), las estudiantes empleaban más estas técnicas que sus compañeros. Por el contrario, no se encontraron diferencias significativas en las escalas de planificación y control; repetición y lectura ni, por tanto, en el uso de las estrategias que las componen. Todo ello demostró que las estudiantes hacían uso de más estrategias asociadas a las fases de adquisición de la información, codificación, recuperación y apoyo; que se esforzaban para sentirse orgullosas de sí mismas; y que antes de empezar a estudiar, distribuían mejor el tiempo. 
En una investigación más reciente realizada con los estudiantes universitarios de distintas carreras (Filosofía, Derecho, Ingeniería Industrial, Informática, Económicas, Empresariales, Biología, Ciencias del trabajo, Educación, Ciencias de la Actividad Física y Deporte) en la Universidad de León (López, 2011), se determinó que la variable sexo producía diferencias significativas en todas las estrategias de aprendizaje salvo en la estrategia de ampliación, donde la diferencia no era significativa entre hombres y mujeres. Este estudio también concluyó que, al observar las diferencias entre género atendiendo a la titulación en curso, la predominancia de las mujeres sobre los hombres como tendencia no se producía del mismo modo para todos los estudios:

$>$ Estudiantes de educación: obtienen mayores puntuaciones en colaboración, conceptualización y participación; menores niveles en estrategias como ampliación y niveles intermedios en preparación de exámenes. Junto con los estudiantes de letras, tienen un mayor uso de estrategias en comparación a los de ciencias.

$>$ Estudiantes de letras: presentan mejores resultados en la escala de ampliación, colaboración y preparación de exámenes (similar a los estudiantes de educación) y menores puntuaciones en estrategias de las escalas de conceptualización y participación (resultados similares a los estudiantes de ciencias). Matiza diferencias entre carreras, por ejemplo, en el caso de los estudiantes de Filosofía solo se encontraron diferencias en las estrategias de colaboración y planificación, y estas favorecían a los hombres en vez de a las mujeres. Esto también sucedía en la carrera de Ciencias del Trabajo.

$>$ Estudiantes de ciencias: son menos estratégicos que el resto, ya que no destaca ninguna estrategia por encima del resto. Además, las diferencias entre las facultades no fueron tan homogéneas. Estudios como Ingeniería industrial e Informática destacaron por utilizar más estrategias de ampliación y los de Biología por las de planificación.

Juárez, Rodríguez y Luna (2012) hallaron que las mujeres hacían un mayor uso de estrategias de aprendizaje y que éste era más variado que el de los hombres, destacando especialmente en las escalas de adquisición, recuperación y apoyo. Ellas utilizaban con mayor frecuencia estrategias como los subrayados, agrupamientos, codificaciones, interacción social, planificación, entre otras, mientras que los estudiantes hacían uso con más frecuencia de secuencias e imágenes. Por último, en una investigación más reciente Jiménez, García, López y Saavedra (2018) demostraron relaciones significativas entre el uso de estrategias por un lado y mayor satisfacción y pensamiento crítico elaborado.

\subsection{Prospectiva de investigación en estrategias de aprendizaje de los estudiantes en modalidad online}

Tal y como ha podido apreciarse a lo largo del punto anterior no existen, hasta ahora, estudios que aborden esta cuestión de investigación más allá de la modalidad presencial. En la tabla 2, mostramos un resumen de las investigaciones realizadas sobre nuestro mismo objeto y que clasificamos en función de si son nacionales o internacionales y del año de publicación. A su vez, se incluyen también los instrumentos de medida utilizados, el objeto de estudio y la muestra, así como las carreras que formaron parte de los estudios y las universidades implicadas.

A modo de síntesis, parece oportuno señalar las siguientes premisas:

$>\quad$ El estudio de las estrategias de aprendizaje y la variable sexo viene siendo estudiada habitualmente junto con otras variables, como por ejemplo el rendimiento o la titulación.

$>$ Las investigaciones realizadas son cuantitativas, siendo ACRA y CHAEA los instrumentos más utilizados.

$>\mathrm{Se}$ detectaron diferencias en función del sexo: ellas generalmente hacían uso de técnicas como subrayados, resúmenes, codificación, presentación y repaso en voz alta; ellos emplearon estrategias de imágenes mentales y autopreguntas.

$>$ Los estudiantes gestionaban mejor la escala ansiedad que las estudiantes.

$>$ Las estudiantes presentaron un mayor rendimiento que los estudiantes.

$>$ En general, las mujeres mostraron en todas las investigaciones el uso de estrategias de todas las escalas (adquisición, codificación, recuperación y apoyo) mientras que los hombres destacaban normalmente en dos (apoyo y codificación). 
Tabla 2. Investigaciones sobre estrategias de aprendizaje y la variable sexo en estudiantes universitarios

\begin{tabular}{|c|c|c|c|c|c|}
\hline INVESTIGACIÓN & INS. & OBJETO DE ESTUDIO & M. & ESTUDIOS & UNIVERSIDAD \\
\hline \multicolumn{6}{|l|}{ Estudios Nacionales } \\
\hline Cano (2000) & $\begin{array}{l}\text { LSI, LASSI, } \\
\text { ASI, ILP }\end{array}$ & $\begin{array}{l}\text { Relación entre género y estrategias de } \\
\text { aprendizaje; género y estilos; género } \\
\text { y procesos de aprendizaje }\end{array}$ & 991 & $\begin{array}{l}\text { Universitarios: Ciencias (Física, Química, } \\
\text { Arquitectura, Medicina), Ciencias Sociales } \\
\text { (Derecho, Psicología, Formación del } \\
\text { profesorado) y Letras (Filología, Historia y } \\
\text { Traducción e Interpretación) }\end{array}$ & Universidad de Granada \\
\hline Martín y Camarero (2001) & $\begin{array}{l}\text { CHAEA, } \\
\text { ACRA }\end{array}$ & $\begin{array}{l}\text { Procesos (estrategias) de aprendizaje, } \\
\text { género y tipo de estudios }\end{array}$ & 1147 & $\begin{array}{c}\text { Universitarios (Informática, Física, Matemáticas, } \\
\text { Derecho, Magisterio) }\end{array}$ & Universidad de Oviedo \\
\hline López (2011) & CETA & $\begin{array}{l}\text { Estrategias de aprendizaje, género, } \\
\text { curso, titulación en curso }\end{array}$ & 805 & $\begin{array}{c}\text { Universitarios: Filosofía, Derecho, Ingeniería } \\
\text { Industrial, Informática, Económicas, } \\
\text { Empresariales, Biología, Ciencias del trabajo, } \\
\text { Educación, FCAFD }\end{array}$ & Universidad de León \\
\hline \multicolumn{6}{|l|}{ Estudios Internacionales } \\
\hline Aguilar (2010) & $\begin{array}{l}\text { CHAEA, } \\
\text { LASSI }\end{array}$ & $\begin{array}{c}\text { Estilos y estrategias de aprendizaje en } \\
\text { universitarios }\end{array}$ & 580 & Universitarios & $\begin{array}{l}\text { Pontífica Universidad } \\
\text { Católicar Argentina } \\
\text { Santa María de Buenos } \\
\text { Aires (Argentina) }\end{array}$ \\
\hline $\begin{array}{l}\text { Rossi, Neer, Lopetegui y } \\
\text { Doná (2010) }\end{array}$ & $\begin{array}{l}\text { ACRA - } \\
\text { Abreviada para } \\
\text { alumnos } \\
\text { universitarios }\end{array}$ & $\begin{array}{l}\text { Estrategias de aprendizaje según el } \\
\text { género. Relación de esas estrategias y } \\
\text { el rendimiento académico }\end{array}$ & 364 & Universitarios & $\begin{array}{l}\text { Universidad Nacional } \\
\text { de La Plata (Argentina) }\end{array}$ \\
\hline $\begin{array}{l}\text { Sepúlveda, Montero, } \\
\text { Pérez, Contreras y Solar } \\
(2010)\end{array}$ & $\begin{array}{l}\text { CHAEA, } \\
\text { ACRA }\end{array}$ & $\begin{array}{c}\text { Diferencias de género, estilos y } \\
\text { estrategias }\end{array}$ & 146 & Farmacología & $\begin{array}{ll}\text { Universidad } & \mathrm{de} \\
\text { Concepción (Chile) } & \end{array}$ \\
\hline $\begin{array}{l}\text { Sepúlveda, López, Torres, } \\
\text { Luengo, Montero y } \\
\text { Contreras (2011) }\end{array}$ & $\begin{array}{l}\text { CHAEA, } \\
\text { ACRA }\end{array}$ & $\begin{array}{l}\text { Estilos y estrategias de aprendizaje, } \\
\text { género, rendimiento }\end{array}$ & 44 & Farmacia, Química & $\begin{array}{ll}\text { Universidad } & \text { de } \\
\text { Concepción (Chile) } & \end{array}$ \\
\hline $\begin{array}{l}\text { Juárez, Rodríguez y Luna } \\
\text { (2012) }\end{array}$ & $\begin{array}{l}\text { CHAEA, } \\
\text { ACRA }\end{array}$ & $\begin{array}{l}\text { Estilos, estrategias de aprendizaje, } \\
\text { titulación y rendimiento }\end{array}$ & 794 & $\begin{array}{c}\text { Universitarios (Psicología, Contaduría, Derecho, } \\
\text { Ingeniería en Computación, Informática } \\
\text { Administrativa, Administración) }\end{array}$ & $\begin{array}{l}\text { Universidad pública del } \\
\text { Estado de México } \\
\text { (México) }\end{array}$ \\
\hline $\begin{array}{l}\text { Jiménez, García, López y } \\
\text { Saavedra (2018) }\end{array}$ & $\begin{array}{c}\text { ACRA } \\
\text { Abreviada }\end{array}$ & Estrategias de aprendizaje & 804 & Grado en Psicología & $\begin{array}{l}\text { Universidad pública de } \\
\text { Andalucía (no concreta) }\end{array}$ \\
\hline
\end{tabular}

Nota: INS=instrumento, M.= muestra. 
$>$ Se identificaron diferencias entre sexos y la titulación en curso, por lo que la variable contextual se postula como moduladora de las diferencias entre hombres y mujeres.

$>$ Los alumnos con mejores calificaciones manifiestan una valoración más positiva de las TIC en los procesos formativos (García y Tejedor, 2017).

\section{MÉTODO}

Llegados a este punto, convenimos en acotar el problema de investigación en el desconocimiento existente en cuanto a las estrategias de aprendizaje y estudiantes universitarios en modalidades distintas a la presencial. El problema de investigación y objetivos aquí descritos forman parte de una investigación desarrollada en el marco de una tesis doctoral. En concreto, exponemos a continuación aquellos aspectos referidos a las estrategias de aprendizaje y la variable sexo, a saber:

$>$ Analizar las estrategias de aprendizaje utilizadas por una muestra de estudiantes que cursan sus estudios siguiendo una modalidad online.

$>$ Identificar diferencias en el uso de estrategias en función del sexo de los y las estudiantes.

$>$ Contrastar semejanzas y diferencias entre los resultados obtenidos y los hallazgos en otras investigaciones en universidades presenciales.

El trabajo de campo lo realizamos siguiendo una metodología mixta. La recogida de datos la realizamos mediante instrumentos cuantitativos (cuestionario) y cualitativos, tales como: (revisión de documentación, observación de aulas virtuales, preguntas abiertas, conversaciones informales, notas de campo...). Posteriormente, el análisis cualitativo efectuado, ha tenido como objetivo favorecer la evaluación e interpretación de los resultados de tipo cuantitativo. Planteamiento construido a partir de Eisner (1998), Erickson (1986) y Woods (1987), quienes mantienen que la investigación cualitativa complementa y resalta la interpretación de resultados, y junto con la propuesta de modelo de secuencia y prioridad de Morgan (1998), este trabajo cualitativo contribuirá a evaluar e interpretar los resultados del trabajo principal y cuantitativo.

La muestra está compuesta por el conjunto de estudiantes del Máster en Formación del Profesorado de Educación Secundaria Obligatoria, Bachillerato, Formación Profesional y Enseñanza de
Idiomas del curso académico 2014-2015 de la Universidad Internacional de Valencia. Los instrumentos y técnicas de recogida de información empleados han sido los siguientes: para el estudio de las estrategias de aprendizaje empleadas por los estudiantes se ha seleccionado el Cuestionario de Evaluación de las Estrategias de Aprendizaje de los Estudiantes Universitarios (CEVEAPEU) de Gargallo, Suárez-Rodríguez y Pérez (2009). Para la recogida de información cualitativa elegimos el análisis documental de reglamentos, memorias e informes de la universidad citada anteriormente, revisión de aulas virtuales y guías didácticas. Al mismo tiempo, la cumplimentación del cuestionario se realizó en un seminario asíncrono en la última de las asignaturas con docencia en el Máster: Innovación e investigación educativa de la especialidad (6 ECTS).

El análisis de datos se realizó con el programa SPSS v17 (Statistical Package Science) para el tratamiento estadístico de los datos cuantitativos (encuesta). En cuanto a los datos cualitativos (la reflexión de los estudiantes acerca de sus estrategias de aprendizaje) se han analizado mediante el uso de la interpretación directa (Stake, 1999) citando algunos de sus relatos con el objetivo de ilustrar y dotar de mayor consistencia a los resultados cuantitativos.

\section{RESULTADOS}

Antes de iniciar el análisis sobre las diferencias entre el sexo de los estudiantes, indicar que la muestra está compuesta por 706 alumnos y alumnas, de los cuales el 59\% son mujeres, 414 estudiantes, mientras que 292 son hombres, lo que representa el 41\%, tal y como se puede ver en la Tabla 3.

Tabla 3. Distribución de los estudiantes según su sexo

\begin{tabular}{cc}
\hline Sexo & N \\
\hline Hombre & 292 \\
Mujer & 414 \\
\hline
\end{tabular}

Nota: $\mathrm{N}=$ número de estudiantes.

Como adelantamos en la concreción de las hipótesis, nuestro propósito es descifrar si existen diferencias en las estrategias de aprendizaje en función del sexo de los estudiantes, por tanto, abordaremos una comparación entre los resultados obtenidos por ambos grupos: hombres y mujeres. Para ello realizaremos un análisis multivariante de la varianza (MANOVA) a través del modelo general lineal, siendo las estrategias de aprendizaje las variables dependientes y el sexo la variable 
independiente. Para estudiar el efecto prestaremos atención al índice Lambda de Wilks y, atendiendo a los resultados obtenidos, determinaremos si el resultado es significativo $(\mathrm{p} \leq 0,05)$. Acto seguido, valoraremos la relevancia a partir del tamaño del efecto, siendo el criterio eta cuadrado parcial $\left(\eta^{2}\right.$ parcial): pequeño $(0,01-0,059)$; mediano $(0,06-$ 0,139); grande (a partir de 0,14) (Cohen, 1977). Y para finalizar, presentaremos los efectos a nivel univariado (ANOVA) para cada una de las 25 estrategias de aprendizaje.

En relación al perfil multivariado y al dominio de las estrategias de aprendizaje entre hombres y mujeres se confirma que existen diferencias altamente significativas en función del sexo según Lambda de Wilks (F29,676=6,415; $\leq \leq 0,000)$ que explica $\left(\eta^{2}\right.$ parcial $\left.=0,216\right)$ un $21,6 \%$ de las diferencias observadas entre los estudiantes, lo que nos permite afirmar que existe un efecto grande o importante (Cohen, 1977). Asimismo (ver Tabla $4^{3}$ ) a nivel univariado existen diferencias significativas $(p \leq$ $0,05)$ en trece de las veinticinco estrategias: motivación intrínseca; valor de la tarea; control de la ansiedad; control, autorregulación; control del contexto; habilidades de interacción social y aprendizaje con compañeros; adquisición de la información; elaboración; organización; almacenamiento, memorización y uso de recursos mnemotécnicos; almacenamiento simple, repetición; transferencia; manejo de recursos para usar la información.

Las diferencias entre los sexos favorecen a las mujeres en estrategias como la motivación intrínseca; valor de la tarea; control, autorregulación; control del contexto; habilidades de interacción social; adquisición de la información; elaboración; organización; almacenamiento, memorización, uso de recursos mnemotécnicos y trasferencia. Sin embargo, presentan menor dominio estratégico para controlar la ansiedad que sus compañeros. Por el contrario, los hombres presentan un nivel estratégico más débil, destacando por encima de las mujeres en la estrategia de almacenamiento simple y repetición.

A su vez, es importante explicar que las puntaciones altas en las estrategias de motivación extrínseca, atribuciones externas, control de la ansiedad y memorización por simple repetición expresan valores estratégicos inadecuados en los estudiantes, por lo que afirmamos que están invertidas respecto al resto de estrategias. Es decir, un valor promedio bajo en éstas será indicador de mejor nivel estratégico (Jiménez, 2015). En nuestro caso, observamos que las puntuaciones son bajas en todas ellas, por lo que el perfil estratégico es adecuado. En cuanto a la relevancia, partiendo del tamaño del efecto $\left(\eta^{2}\right.$ parcial $)$ todas las estrategias con resultados significativos tienen un tamaño pequeño al situarse entre 0,01 - 0,059 (Cohen, 1977) a excepción de la estrategia de elaboración con un efecto mediano, lo que la variable sexo explicaría el $7,5 \%$ de las diferencias en dicha variable.

\footnotetext{
3 La Tabla 4 muestra únicamente las estrategias con resultados significativos.
} 
Tabla 4. ANOVA, estadísticos descriptivos, F univariada y significación de estrategias de aprendizaje en relación al sexo de los estudiantes

\begin{tabular}{|c|c|c|c|c|c|c|}
\hline Estrategias & Sexo & Media & $\begin{array}{l}\text { Desviación } \\
\text { típica }\end{array}$ & $\mathbf{F}$ & Sig. & $\eta_{\text {parcial }}^{2}$ \\
\hline Motivación intrínseca & $\begin{array}{l}\text { Hombre } \\
\text { Mujer }\end{array}$ & $\begin{array}{l}4,2283 \\
4,3897\end{array}$ & $\begin{array}{l}, 54328 \\
, 50719\end{array}$ & 16,341 &, 000 &, 023 \\
\hline Valor de la tarea & $\begin{array}{l}\text { Hombre } \\
\text { Mujer }\end{array}$ & $\begin{array}{l}4,2637 \\
4,3545\end{array}$ & $\begin{array}{l}48143 \\
, 50041\end{array}$ & 5,813 &, 016 & ,008 \\
\hline Control ansiedad & $\begin{array}{l}\text { Hombre } \\
\text { Mujer }\end{array}$ & $\begin{array}{l}2,8673 \\
2,9577\end{array}$ & $\begin{array}{l}, 51746 \\
, 52339\end{array}$ & 5,160 & ,023 & ,007 \\
\hline Control, autorregulación & $\begin{array}{l}\text { Hombre } \\
\text { Mujer }\end{array}$ & $\begin{array}{l}3,9127 \\
4,0346\end{array}$ & $\begin{array}{l}48329 \\
, 43645\end{array}$ & 12,226 &, 001 &, 017 \\
\hline Control del contexto & $\begin{array}{l}\text { Hombre } \\
\text { Mujer }\end{array}$ & $\begin{array}{l}3,9264 \\
4,1045\end{array}$ & $\begin{array}{l}61564 \\
, 61197\end{array}$ & 14,431 &, 000 &, 020 \\
\hline $\begin{array}{l}\text { Habilidades de interacción social y aprendizaje } \\
\text { con compañeros }\end{array}$ & $\begin{array}{l}\text { Hombre } \\
\text { Mujer }\end{array}$ & $\begin{array}{l}3,5634 \\
3,8007\end{array}$ & $\begin{array}{l}, 80299 \\
, 83431\end{array}$ & 14,296 &, 000 & ,020 \\
\hline Adquisición de la información & $\begin{array}{l}\text { Hombre } \\
\text { Mujer }\end{array}$ & $\begin{array}{l}3,5879 \\
3,7713\end{array}$ & $\begin{array}{l}, 71814 \\
, 72897\end{array}$ & 10,976 &, 001 &, 015 \\
\hline Elaboración & $\begin{array}{l}\text { Hombre } \\
\text { Mujer }\end{array}$ & $\begin{array}{l}4,0950 \\
4,3744\end{array}$ & $\begin{array}{l}51789 \\
, 46053\end{array}$ & 56,795 &, 000 &, 075 \\
\hline Organización & $\begin{array}{l}\text { Hombre } \\
\text { Mujer }\end{array}$ & $\begin{array}{l}3,7185 \\
4,0411\end{array}$ & $\begin{array}{l}, 79867 \\
, 72823\end{array}$ & 30,998 &, 000 &, 042 \\
\hline $\begin{array}{l}\text { Almacenamiento, memorización, uso de } \\
\text { recursos mnemotécnicos }\end{array}$ & $\begin{array}{l}\text { Hombre } \\
\text { Mujer }\end{array}$ & $\begin{array}{l}3,5114 \\
3,8205\end{array}$ & $\begin{array}{l}, 88616 \\
, 83263\end{array}$ & 22,361 &, 000 &, 031 \\
\hline Almacenamiento simple, repetición & $\begin{array}{l}\text { Hombre } \\
\text { Mujer }\end{array}$ & $\begin{array}{l}1,9640 \\
1,7162\end{array}$ & $\begin{array}{l}, 80243 \\
, 68872\end{array}$ & 19,322 &, 000 &, 027 \\
\hline Transferencia & $\begin{array}{l}\text { Hombre } \\
\text { Mujer }\end{array}$ & $\begin{array}{l}4,1370 \\
4,2882\end{array}$ & $\begin{array}{l}, 50302 \\
., 51043\end{array}$ & 15,218 &, 000 &, 021 \\
\hline $\begin{array}{l}\text { Manejo de recursos para usar la información } \\
\text { adquirida }\end{array}$ & $\begin{array}{l}\text { Hombre } \\
\text { Mujer }\end{array}$ & $\begin{array}{l}3,8408 \\
3,9541\end{array}$ & $\begin{array}{l}, 70429 \\
, 70045\end{array}$ & 4,464 &, 035 & ,006 \\
\hline
\end{tabular}

Para finalizar, tal y como observamos en la figura 1, las mujeres presentan un mejor patrón estratégico tanto en las estrategias que componen la escala afectiva, de apoyo y control (motivación, componentes afectivos, estrategias metacognitivas, estrategias de control y relación con el contexto) como en las estrategias relacionadas con el procesamiento de la información (búsqueda, selección y procesamiento). En el caso de las últimas, las diferencias existentes son mayores en seis de las ocho estrategias, apreciándose con más claridad un mejor perfil estratégico relacionado con el procesamiento de la información en las mujeres que en los hombres. Además, debemos tener presente que la estrategia de almacenamiento simple, estrategia con valor invertido, corrobora esta explicación.

Deteniéndonos en cada estrategia, en la subescala de estrategias motivacionales, las mujeres presentan mejor dominio en las estrategias de motivación intrínseca, valor de la tarea y atribuciones internas. En este sentido, algunas de las opiniones manifestadas por las estudiantes corroboran los resultados obtenidos en relación a dichas estrategias tal y como queda reflejado en las siguientes citas:

Tengo una motivación interna que me permite apreciar más lo que estudio y sentirme bien con lo que hago (Estudiante 250, 2-3).

(...) mi motivación no reside en factores externos, si no en valores intrínsecos y en el propio deseo de aprender más que en otros elementos como el estatus, las influencias, etc. (Estudiante 163, 6-8).

(...) las estrategias motivacionales que me caracterizan como alumna, a día de hoy, son infinitamente más sanas, maduras y coherentes que años atrás (...) (Estudiante 224, 24-25). 


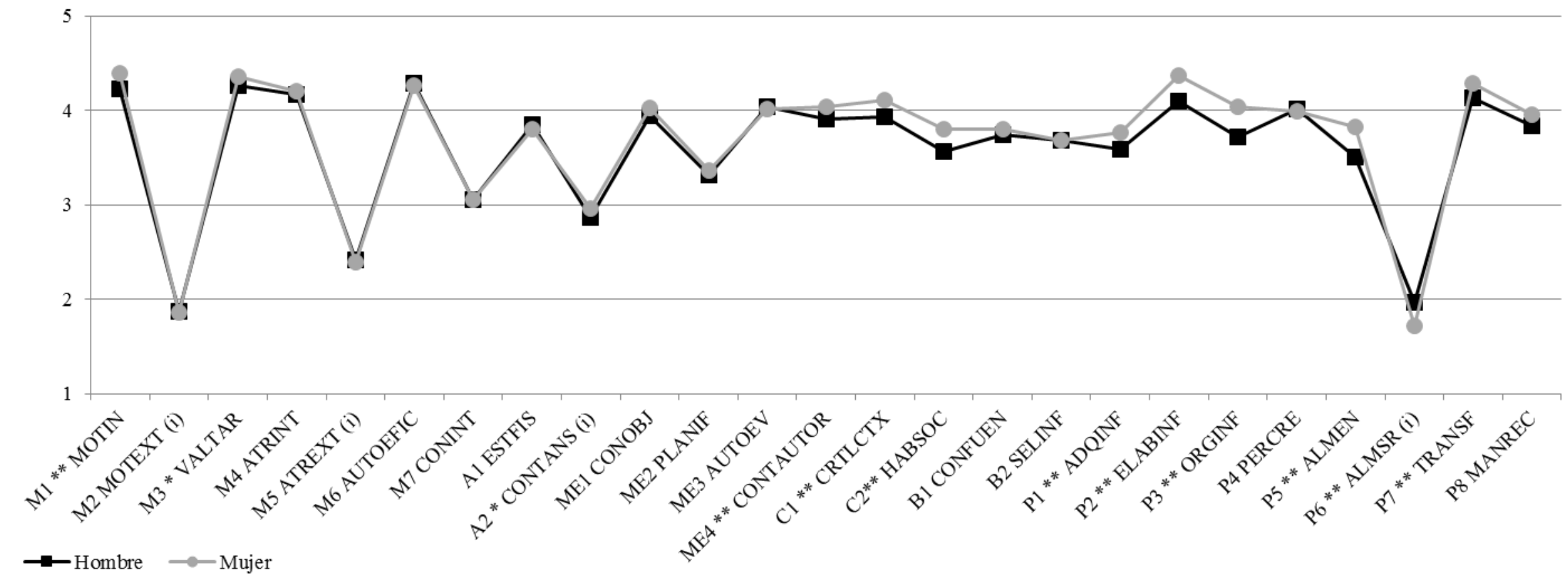

Figura 1. Diferencias en estrategias de aprendizaje en función del sexo. Nota: M1 a M7 estrategias pertenecientes a la subescala motivacional: Motivación intrínseca (MOTIN); Motivación extrínseca (MOTEXT); Valor de la tarea (VALTAR); Atribuciones internas (ATRINT); Atribuciones externas (ATREXT); Autoeficacia y expectativas (AUTOEFIC); Inteligencia como modificable (CONINT); A1 y A2, subescala afectiva: Estado físico y anímico (ESTFIS); Control ansiedad (CONTANS); ME1

a ME4 subescala metacognitiva: Conocimiento objetivos y criterios de evaluación (CONOBJ); Planificación (PLANIF); Autoevaluación (AUTOEV); Control,

autorregulación (CONTAUTOR); C1 y C2 subescala contexto: Control del contexto (CRTLCTX); Habilidades de interacción social y aprendizaje con compañeros

(HABSOC); B1 y B2 subescala búsqueda y selección de información; Conocimiento de fuentes y búsqueda de información (CONFUEN); Selección de información

(SELINF); P1 a P8 subescala procesamiento: Adquisición de la información (ADQINF); Elaboración (ELABINF); Organización (ORGINF); Personalización y creatividad, pensamiento crítico (PERCRE); Almacenamiento, memorización, uso de recursos mnemotécnicos (ALMEN); Almacenamiento simple, repetición (ALMSR); Transferencia (TRANSF); Manejo de recursos para usar la información adquirida (MANREC). ${ }^{* *}$ ) Nivel de significación $p \leq 0,01$. (*) Nivel de significación $p \leq 0,05$. (i) Estrategias invertidas. 
Por otro lado, los hombres obtuvieron puntuaciones más altas en cuatro de las ocho estrategias que componen la misma subescala, dos de ellas variables invertidas: motivación extrínseca, atribuciones externas, autoeficacia y expectativas y concepción de la inteligencia como modificable. Resulta revelador el testimonio del siguiente estudiante sobre la motivación extrínseca:

(...) en mi caso existe una gran dominante de la motivación extrínseca producida en parte a que llevo a cabo el estudio para no defraudar a mis familiares, (...) también observo cómo me resulta necesario que otras personas, como padres, amigos, profesores, me animen y me motiven a estudiar. (Estudiante 611, 2-7)

En la subescala de componentes afectivos las puntuaciones fueron muy similares entre hombres y mujeres, siendo los hombres los que estuvieron levemente por encima en las estrategias de estado físico y anímico positivo y las mujeres en control de la ansiedad, estrategia invertida en la que las puntuaciones altas indicarían un comportamiento poco recomendable. Sobre este aspecto las estudiantes opinaron lo siguiente:

(...) la ansiedad es y fue uno de mis peores amigos durante mis estudios, el hablar en público y los nervios previos a un examen era uno de mis peores momentos, algo que intento solucionar poco a poco. (Estudiante 64, 34-36)

(...) cuesta mucho relajarse y estar tranquila ante exámenes, exposiciones y demás. Ya que tiendo a identificar esos fracasos como una falta de superación a nivel personal. (Estudiante 335, 1719)

(...) en cuanto me pongo frente a un examen sigo poniéndome nerviosa y una vez realizado el examen siempre tiendo a darle vueltas a lo que ya he hecho. (Estudiante 382, 19-21)

En cuanto a la subescala de estrategias metacognitivas, a excepción de la estrategia de autoevaluación en la que los hombres puntuaron ligeramente por encima, las mujeres los superaron en las estrategias de conocimiento de objetivos y criterios de evaluación, autoevaluación y control y regulación del contexto. Como hicimos con las estrategias anteriores, nos ha parecido relevante recoger dos citas con el propósito de ilustrar la visión de los estudiantes:

(...) tengo buena capacidad de autoevaluación sobre mis puntos fuertes y débiles, lo que me lleva a un decente autocontrol e improvisación sobre la marcha para adaptarme a distintos contextos y profesores. (Estudiante 174, 31-33)

Es muy importante el control y la autorregulación, porque debemos adaptarnos a las asignaturas y a los profesores, y yo personalmente cambio mi idea de estudio cuando pienso que es necesario, (...) debemos planificar nuestra manera de aprender en las asignaturas y qué necesita cada profesor en su asignatura, dedicando más tiempo si es necesario a las asignaturas más difíciles o aquellas que entendemos menos. (Estudiante 129, 39-45)

En la última de las escalas de estrategias afectivas, apoyo y control, es decir, la subescala de estrategias de control del contexto, interacción social y manejo de recursos se observa que las mujeres obtuvieron mejores puntuaciones en ambas estrategias, control del contexto y habilidades sociales. De hecho, hasta el momento son las estrategias en las que se aprecian más claramente las diferencias entre sexos. La reflexión de la siguiente estudiante da muestra de ello:

Mi lugar de estudio es adecuado y no pierdo el tiempo cuando se trata de estudiar (...) considero muy importante y de gran ayuda saber trabajar en equipo (...) los compañeros dan puntos de vista diferentes al que uno tiene, resuelven dudas. Además, suelo escoger compañeros igual de responsables que yo para poder trabajar en armonía (...). (Estudiante 213, 55-62)

Como adelantamos anteriormente, es en la escala de estrategias relacionadas con el procesamiento de la información en la que se aprecia con más claridad el perfil estratégico de las mujeres: ellas obtienen mejor media que los hombres (y con mayor claridad) en las estrategias de conocimiento de fuentes, búsqueda de la información y selección de la información (primera subescala); adquisición de la información, elaboración, organización, almacenamiento y uso de recursos mnemotécnicos, transferencia y manejo de recursos.

El pensamiento crítico ha sido uno de los pilares, no solo de mis estudios, sino de mí día a día; no interiorizo ninguna información sin estar fundamentada en un ámbito científico o sociocultural. (Estudiante 661, 33-35)

Siempre realizo esquemas, subrayo apuntes, hago resúmenes y selecciono la información más relevante y que considero más importante de la asignatura (...) suelo ampliar información 
buscando noticias de actualidad, apuntes, esquemas. Utilizo mucho Internet para la búsqueda de esta información. (Estudiante 477, 64-72)

Por el contrario, los hombres obtuvieron mejor puntuación (con escasa diferencia en comparación con sus compañeras) en personalización, creatividad, pensamiento crítico y en almacenamiento simple y repetición, de nuevo una estrategia invertida.

\section{DISCUSIÓN}

Con el propósito de no resultar iterativo este apartado con el de resultados, en el que ya pudieron vislumbrarse las diferencias entre sexos, pasamos a resaltar los hallazgos de nuestro estudio cotejándolos con las averiguaciones de otras investigaciones sobre universidades presenciales.

En primer lugar, los resultados del perfil multivariado (MANOVA) y dominio de estrategias entre sexos se confirmaron al obtener diferencias altamente significativas según Lambda de Wilks (F29,676 $=6,415 ; \mathrm{p} \leq 0,000)$ que explicó un $21,6 \%$ $\left(\eta^{2}\right.$ parcial $\left.=0,216\right)$ de las diferencias observadas entre los estudiantes, lo que se considera un efecto grande o importante (Cohen, 1977). Asimismo, a nivel univariado se lograron diferencias muy significativas $(p \leq 0,01)$ en diez estrategias (motivación intrínseca; autorregulación; control del contexto; habilidades de interacción social; adquisición de la información; elaboración; organización; almacenamiento, memorización, uso de recursos mnemotécnicos y trasferencia) y significativas $(\mathrm{p} \leq 0,05)$ en dos (valor de la tarea y control de la ansiedad). Dichos resultados quedaron reflejados en la Tabla 4. Esto nos permite afirmar que los hombres presentan un perfil estratégico más débil en comparación al de las mujeres, hecho que pudimos observar claramente en la Figura 1, especialmente en las subescalas de estrategias de control de contexto, interacción social y manejo de recursos; y en la de estrategias de búsqueda, selección, procesamiento y uso de la información.

Los resultados concuerdan con los alcanzados en investigaciones tales como las de Aguilar (2010), Cano (2000), Martin y Camarero (2001), Lopes (2013) y Sepúlveda et al. (2010, 2011), en los que las estudiantes destacaron en estrategias como búsqueda de codificaciones, elaboración de esquemas o resúmenes, uso de acrónimos y palabras clave y planificación en la respuesta, ayudas al estudio y repaso de lo aprendido, entre otras, todas ellas estrategias relacionadas con el procesamiento de la información. En cuanto al uso de estrategias afectivas y de control, en dichos estudios las alumnas destacaban en actitud, motivación en el estudio, administración del tiempo etc., comportamiento que se corrobora también en nuestro caso: ellas planifican y aprovechan más el tiempo de estudio, presentan una motivación intrínseca por el aprendizaje, tienen una marcada preferencia por realizar trabajos y estudiar con otros compañeros, comentar dudas y pedir ayuda tanto a sus iguales como al profesor.

En cuanto a la estrategia de control de la ansiedad, estrategia invertida en la que las mujeres obtuvieron peor resultado, indicar que los resultados son similares a los obtenidos en las investigaciones de Aguilar (2010) y Cano (2000). Y aunque las estudiantes deben mejorar en ese aspecto, los resultados en el resto de las estrategias, como adelantamos anteriormente, corroboran que hacen mejor uso de las estrategias de aprendizaje que sus compañeros. En concreto, ellas afirmaron ponerse nerviosas ante exámenes o tener que hablar en público, mientras que ellos afirmaron poder relajarse ante este tipo de situaciones en las que hay un mayor estrés.

Como conclusión se apreciaron diferencias significativas entre los alumnos y alumnas, concretándose un mejor perfil estratégico en el caso de las mujeres en comparación al de los hombres: las estudiantes obtuvieron puntuaciones medias más elevadas en 67 de los 88 ítems que forman parte del cuestionario. A tenor de estos resultados, a nuestro entender, habría que seguir indagando respecto a si estas diferencias y en cómo enfrentan las situaciones de aprendizaje, se consolidan o tienden a desaparecer. Propósito de investigación que además de nuestros datos de campo, se ampara en el derecho a la igualdad de oportunidades con independencia del sexo.

Igualmente, una propuesta de actuación en los estudiantes universitarios online debería ir encaminada necesariamente tanto a la mejora del conocimiento en sí como en la profundización de estrategias avanzadas de búsqueda y evaluación de información. En una sociedad inminentemente digital, independientemente del sexo, los esfuerzos han de centrarse en las áreas de información y alfabetización. Es por este motivo por el que se proponen talleres prácticos en los que el alumnado aprenda las competencias necesarias para de identificar, localizar, recuperar, almacenar y analizar información digital así como ser capaces de evaluar su finalidad y relevancia. 


\section{REFERENCIAS}

Aguilar, Ma C. (2010). Estilos y estrategias de aprendizaje en jóvenes ingresantes a la universidad. Revista de Psicología, 28(2), 208-226.

Alonso, C., Gallego, D. y Honey, P. (1994). Los estilos de aprendizaje. Procedimientos de diagnóstico y mejora. Bilbao: Ediciones Mensajero.

Amelang, J. S. y Nash, M. (1990). Historia y género: las mujeres en la Europa moderna y contemporánea. Valencia: Edicions Alfons el Magnànim, Institució Valenciana d'Estudis i Investigació.

Bahamón, J., Vianchá, M. A. y Alarcón, L. L. (2012). Estilos y estrategias de aprendizaje: una revisión empírica y conceptual de los últimos diez años. Pensamiento Psicológico, 10(1), 129-144.

Bates, T. (2001). Cómo gestionar el cambio tecnológico: estrategias para los responsables de centros universitarios. Barcelona: Gedisa.

Bourdieu, P. (2000). La dominación masculina. Barcelona: Anagrama.

Cabero, J., Fernández, B. y Marín, V. (2017). Dispositivos móviles y realidad aumentada en el aprendizaje del alumnado universitario. RIED. Revista Iberoamericana de Educación a Distancia, 20(2), 167-185.

Cano, F. (2000). Diferencias de género en estrategias y estilos de aprendizaje. Psicothema, 12(3), 360-367.

Cohen, (1977). Statistical power analysis for behavioral sciences. New York: Academic Press.

Da Cuña, I., Gutiérrez, M., Barón, F. J. y Labajos, Mª T. (2014). Influencia del nivel educativo de los padres en el rendimiento académico, las estrategias de aprendizaje y los estilos de aprendizaje, desde la perspectiva de género. Revista de estilos de aprendizaje, 7(13), 64-84.

Eisner, E. W. (1998). El ojo ilustrado. Indagación cualitativa y mejora de la práctica educativa. Barcelona: Paidós.

Erickson, F. (1986). Qualitative methods in research on teaching. En M. C. Wittrock (Eds.) Handbook of research on teaching (pp. 119-159). Nueva York: Macmillan.

García, A, y Tejedor, F. J. (2017). Percepción de los estudiantes sobre el valor de las TIC en sus estrategias de aprendizaje y su relación con el rendimiento. Educación XXXI, 20(2), 137-159.

García, A. (2011). Efectos del estrés percibido y las estrategias de aprendizaje cognitivas en el rendimiento académico de estudiantes universitarios noveles de ciencias de la salud. Málaga: Universidad de Málaga.

García, L. (2001). La educación a distancia. De la teoría a la práctica. Barcelona: Ariel.

Gargallo, B., Suárez, J., Pérez, C. (2009). El cuestionario CEVEAPEU. Un instrumento para la evaluación de las estrategias de aprendizaje de los estudiantes universitarios. Relieve, 15(2), 1-31.

Gómez, M. (2018). La realidad aumentada, los códigos QR y la geolocalización móvil para educación. En M. L. Cacheiro (Coord.) Educación y tecnología: estrategias didácticas para la integración de las TIC, Madrid: Universidad Nacional de Educación a Distancia.

Jiménez, L., García, A. J., López, J. y Saavedra, F. J. (2018). Evaluación de estrategias de aprendizaje mediante la escala ACRA abreviada para estudiantes universitarios. Revista Psicodidáctica, 23(1), 63-69.

Jiménez, R. (2015). Análisis de los procesos de aprendizaje y el rendimiento académico de los estudiantes universitarios y su relación con dimensiones personales y contextuales
(Tesis Doctoral Inédita). Valencia: Universitat de València.

Juárez, C., Rodríguez, G. y Luna, E. (2012). El cuestionario de estilos de aprendizaje CHAEA y la escala de estrategias de aprendizaje ACRA como herramienta potencial para la tutoría académica. Revista de Estilos de Aprendizaje, 10(10), 148-171.

Kite, M. (2001). Gender Stereitypes. En J. Worell (Eds.), Encyclopedia of Women and Gender: Sex Similarities and Differences and the Impact of Society on Gender (pp. 561-570). London: Academic Press.

Lópes, P. (2013). Análisis Multivariante de la relación entre Estilos/Estrategias de Aprendizaje $e$ Inteligencia Emocional, en alumnos de Educación Superior (Tesis Doctoral Inédita). Salamanca: Universidad de Salamanca.

López, M. (2011). Estrategias de aprendizaje en estudiantes universitarios. Diferencias por género, curso y tipo de titulación. Revista Teoría de la Educación: Educación y Cultura en la Sociedad de la Información, 12(2), 203233.

Martín, F. y Camarero, F. (2001). Diferencias de género en los procesos de aprendizaje universitarios. Psicothema, 13(4), 598-604.

Morgan, D. (1998). Practical strategies for combining qualitative and quantitative methods: applications to health research. Qualitative Health Research, 8(3), 362376

Raftopoulos, M. (2014). Towards gamification transparency: A conceptual framework for the development of responsible gamified enterprise systems. Journal of Gaming \& Virtual Worlds, 6(2), 159-178.

Román, J. M. y Gallego, S. (1994). ACRA. Escalas de estrategias de aprendizaje. Madrid: TEA.

Rossi, L.E., Neer, R.H., Lopetegui, M.S. y Doná, S. (2010). Estrategias de aprendizaje y rendimiento académico según el género en estudiantes universitarios. SRevista de Psicología, 11, 199-211.

San Martín, A., Peirats, J. y Sales, C. (2006). Controversias en torno al software libre: prácticas y discursos en la docencia universitaria valenciana. Revista Iberoamericana de Educación a Distancia (RIED), 11(1), 135-156.

Sánchez, A., Conde, E., Meroño, L., Arias, J. L., García, J. A. (2018). Estudio del éxito académico de un modelo universitario de carrera dual en deportistas-estudiantes según género, nivel de estudios y deporte. Revista española de educación física y deportes-REEFD-, 421, $35-47$.

Scott, J. W. (1986). Gender: A Useful Category of Historical Analysis. The American Historical Review, 91(5), 10531075.

Sepúlveda, Mª J., López, M., Torres, P., Luengo, J., Montero, E. y Contreras, E. (2011). Diferencias de género en el rendimiento académico y en el perfil de estilos y de estrategias de aprendizaje en estudiantes de química y farmacia de la Universidad de Concepción. Revista Estilos de Aprendizaje, 7(7), 135-150.

Sepúlveda, Ma J., Montero, E., Pérez, R., Contreras, E. y Solar, $M^{a}$ I. (2010). Diferencias de género en el perfil de estilos y del uso de estrategias cognitivas de aprendizaje de estudiantes de Farmacología. Revista Estilos de Aprendizaje, 5(5), 63-83.

Stake, R. E. (1999). Investigación en estudio de casos. Madrid: Morata.

Woods, P. (1987). La escuela por dentro. La etnografía en la investigación educativa. Barcelona: Paidós. 